\title{
Deep eutectic solvents comprising creatine and citric acid and their hydrated mixtures
}

\author{
Matthew P. Heaney, ${ }^{a}$ Laxmi Adhikari, ${ }^{b}$ Asher L. Siegel, ${ }^{b}$ Kyle B Pekar, ${ }^{\text {a } J o n a t h a n ~ B ~ L e f t o n, ~}{ }^{\text {a }}$ Claire \\ Lamberti, ${ }^{a}$ Pokpong Rungthanaphatsophon, ${ }^{b}$ Justin R. Walensky, ${ }^{b}$ Gary A. Baker*b and Tomče \\ Runčevski*a
}

\begin{abstract}
We report the phase diagram for the binary creatine-citric acid mixture which features a stable and broad eutectic region. Combinations containing $\mathbf{1 0}-\mathbf{6 0} \mathrm{mol} \%$ creatine yield a deep eutectic solvent with a glass transition temperature at $270 \mathrm{~K}$. Addition of up to $70 \mathrm{~mol} \%$ water to the binary mixture affords retention of the eutectic nature and a handle to vary solvent viscosity and polarity.
\end{abstract}

Deep eutectic solvents (DES) are a unique class of materials that consist of a particular mixture of organic compounds which drastically lowers the system's melting point, well below that of each pure constituent. In the past few decades, these materials have garnered attention as sustainable solvents in catalysis, extractions, separations, cryopreservation, and myriad other applications. ${ }^{1}$ To represent truly green solvents, DESs should incorporate biocompatible and non-toxic components. There has been significant progress in designing such DESs, ${ }^{2}$ however, further research remains necessary. Herein, we present a new subtype of benign and inherently edible DESs composed of creatine ( $\mathrm{CrA})$ and citric acid (CAA, Scheme 1). Typically, mixing two organic components leads to a gradual decrease in melting point that converges on a single eutectic point. Congruent melting is usually observed at the eutectic point, with different compositions generating solid-liquid multi-phase systems. The occurrence of a solid phase impedes typical applications of a DES. As such, we sought to address this challenge by designing a novel DES which features a broad eutectic halt, tuneable chemical composition/physical properties, and generalized applicability across multiple fields.

Creatine, $\mathrm{N}$-(aminoiminomethyl)- $\mathrm{N}$-methyl glycine, is a naturally produced compound involved in energetic shuttling during strenuous exercise. Its popularization as an ergogenic aid in the supplement industry is owed to the significant enhancement of both anaerobic exercise capacity and maximal strength performance reported in athletes. ${ }^{3}$ This traction has continued into the present, creating a market valued at \$350-550 million in the U.S. alone. Currently, there are two formulations on the market: creatine monohydrate and creatine hydrochloride. Recently, we reported a mechanochemical route to the preparation of coamorphous and cocrystalline formulations of creatine and citric acid with

\footnotetext{
a. Department of Chemistry, Southern Methodist University, Dallas, TX 75275 (USA) E-mail: truncevski@smu.edu

b. Department of Chemistry, University of Missouri, Columbia, MS 65211 (USA)

E-mail: bakergar@missouri.edu
}<smiles>CN(CC(=O)O)C(=N)N</smiles><smiles>O=C(O)CC(O)(CC(=O)O)C(=O)O</smiles>

Scheme 1. Structural formulas of creatine ( $\mathrm{CrA}$ ) and citric acid (CAA).

improved solubility and decreased acidity. ${ }^{4}$ Motivated by the observation of a coamorphous solid, the extensive hydrogen bonding in the cocrystal, and the propensity of citric acid towards the formation of DESs ${ }^{5}$, glasses, and amorphous solids, ${ }^{6}$ we explored the possibility of DES innovation using creatine and citric acid components with edible or nutritive qualities.

To explore the solid-liquid phase boundaries and the thermodynamic characteristics of the binary system of creatine and citric acid, a composition-temperature $(\chi-T)$ phase diagram was constructed, as shown in Fig. 1. We observed the exo- and endothermic peak maxima of the heat flux in the differential scanning calorimetry (DSC) curves collected upon heating and cooling of samples with varying component molar ratios (Fig. S1 in the ESI). Heating of a physical mixture of creatine and citric acid resulted in melting at $363 \mathrm{~K}$, a temperature significantly

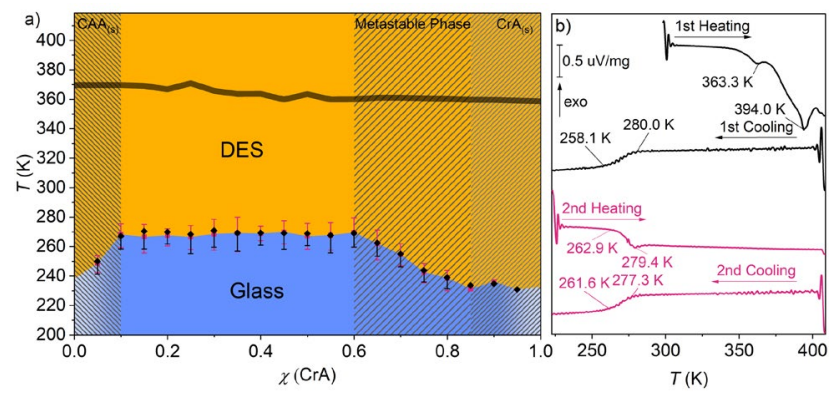

Fig. 1 a) Binary $\chi-T$ phase diagram for the creatine (CrA)-citric acid (CAA) system. The glass transition temperatures are presented in black and red dots, as detected in the first and second heating cooling cycles, respectively. The hatched regions indicate coexistence of a crystalline phase. The temperature of preparation (melting of physical mixture of creatine and citric acid) is denoted by the thick gray line. b) Representive DSC curves collected for a $\chi(\mathrm{CrA})$ $=0.45$ sample.

below the decomposition (without melting) of pure anhydrous creatine $(528 \mathrm{~K})$ or the melting of pure citric acid $(426 \mathrm{~K})$. Cooling of the so-formed DES results in glass formation with a glass transition temperature $\left(T_{\mathrm{g}}\right)$ with an onset near $270 \mathrm{~K}$. As 
anticipated, the phase composition and thermodynamic properties were found to depend upon the constituent molar ratios. Heating of samples having an anhydrous creatine mole fraction, $\chi(\mathrm{CrA})$, in the range of $c a$. $0-0.10$ resulted in a twophase system of solid anhydrous citric acid and DES. Samples in the range of $\chi(\mathrm{CrA}) \approx 0.10-0.60$ yielded a well-behaved, singlephase DES. These DESs were found to be stable for months when stored in closed containers. Within this compositional range, all DESs exhibited a $T_{\mathrm{g}}$ at $\sim 270 \mathrm{~K}$. Samples in the range of $\chi(\mathrm{CrA}) \approx 0.6-0.85$ gave metastable DESs, as indicated by the sporadic crystallization of anhydrous creatine that typically followed within days. Interestingly, the $T_{\mathrm{g}}$ of these metastable DESs showed an inverse and linear dependence upon increasing $\chi(\mathrm{CrA})$. Finally, samples for $\chi(\mathrm{CrA})>0.85$ featured two phases: solid anhydrous creatine and a DES phase. Across different compositions, the thermal decomposition of the DESs begins at $\sim 420 \mathrm{~K}$ (Fig. S2 in the ESI). Taken together, these results establish the $\chi(\mathrm{CrA}) \approx 0.10-0.60$ and $270-420 \mathrm{~K}$ region in the phase diagram as a compositional and thermal range of stability of single-phase DESs composed of creatine and citric acid.

The creatine-citric acid DES exhibits very high viscosity at room temperature, estimated to be greater than $10^{6} \mathrm{cP}$ (vide infra). Highly viscous solvents and liquids have various practical applications, particularly in polymer synthesis. In fact, the biocompatible nature of creatine and citric acid makes this family of DESs particularly intriguing for biomedical applications. For example, they may have use for the cryopreservation of viruses and proteins or as an adhesive medium for the topical delivery of various medicines. Finally, this highly viscous material can be marketed as an entirely new formulation for creatine supplementation with major advantages over the commercially available crystalline counterparts, particularly regarding taste, processability, and aqueous solubility. ${ }^{4}$

We also recognize that the high viscosity limits certain applications as a solvent or reaction medium. One way to overcome this drawback is the incremental addition of a compatible cosolvent, such as water. ${ }^{7}$ Small amounts of added water become an integral part of the DES, incorporating within the local structure and engaging in intermolecular hydrogen bonding between creatine and citric acid molecules. Expectedly, the presence of water and the dynamic intermolecular interactions lead to a significant decrease in viscosity. However, this strategy has a critical intrinsic limitation. Adding large amounts of water to the system will eventually destroy the integrity of the DES and lead to the formation of an aqueous solution of creatine and citric acid. As such, we sought to answer the important question, "how much water can be added before the DES phase dissociates?" by systematic investigation of the phase diagram.

To construct the $\chi-T$ phase diagram of $1: 1$ creatine:citric acid (Fig. 2a) with water added as a third component, we observed the peak maxima of the heat flux in the DSC curves collected upon heating and cooling below room temperature (Fig. S3 in the ESI). Anhydrous DES undergoes typical vitrification with a glass transition temperature of $270 \mathrm{~K}$ (Fig. S1 in the ESI). The gradual addition of water results in a linear decrease in the

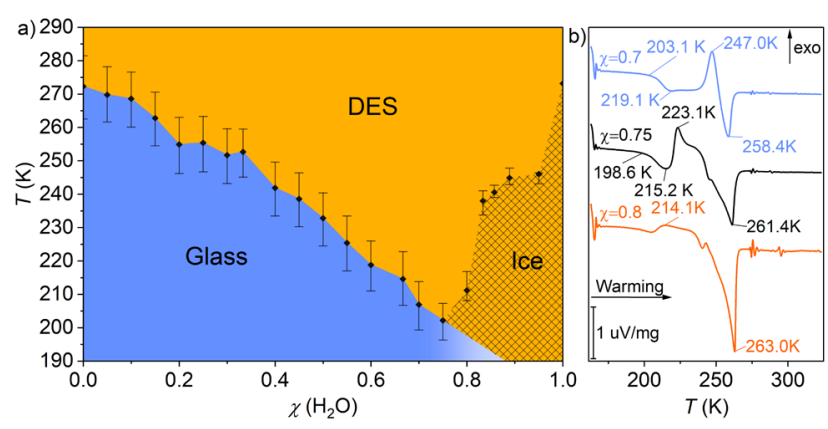

Fig. 2 a) $\chi-T$ phase diagram of the binary $1: 1$ creatine:citric acid system with water added as a third component. The hatched regions denote detection of a crystalline phase. The $T_{\mathrm{g}}$ and ice melting values are presented as black dots, as detected in the heating cycle. b) Example of DSC curves, where cold crystallization is observed, collected on samples for $\chi\left(\mathrm{H}_{2} \mathrm{O}\right)=0.70,0.75$, and 0.80 .

$T_{\mathrm{g}}$ to a minimum value of $\sim 200 \mathrm{~K}$ at $\chi\left(\mathrm{H}_{2} \mathrm{O}\right)=0.75(75 \mathrm{~mol} \%$ water, equivalent to an $\mathrm{CrA}: \mathrm{CAA}: \mathrm{H}_{2} \mathrm{O}$ ratio of $\left.12.5: 12.5: 75\right)$. Further increase in the water content leads to water-ice formation, evidenced by sharp exothermic and endothermic peaks upon cooling and heating, respectively (Fig. S3 in the ESI). Based on these observations, we hypothesize that below 75 mol\% water, the water molecules are an integral part of the DES and, upon cooling, the mixture solidifies as a homogeneous, single-phase amorphous solid. Above 75 mol\% water, the water molecules interact with one another in a bulk liquid phase that dissolves creatine and citric acid. As a result, the formation of ice is observed upon cooling. Significantly, in addition to decreasing the viscosity, addition of water to 1:1 CrA:CAA also extends the lower boundary of the thermal stability region an additional $\sim 70 \mathrm{~K}$.

The system's transition from a DES to an aqueous solution is gradual and gives rise to a distinctive concentration region where water molecules form aqueous solution nanoclusters. This region features unique thermophysical properties, such as thawing crystallization. ${ }^{8}$ This phenomenon has received special attention as it can cause osmotic stress and severe mechanical damage to cryopreserved biological samples and living organisms. ${ }^{8}$ Fig. 2b presents DSC curves for 1:1 CrA:CAA samples for $\chi\left(\mathrm{H}_{2} \mathrm{O}\right)=0.70-0.80$. For these samples, the first thermal event upon heating is a glass transition temperature at 214-219 K. This endothermic process generates a localized cooling of the system, which subsequently prompts ice nucleation and crystallization of water nanoclusters. This is evidenced by a sharp exothermic crystallization peak in the DSC curve at 247 and $223 \mathrm{~K}$ for the $\chi\left(\mathrm{H}_{2} \mathrm{O}\right)=0.70$ and 0.75 samples, respectively. Further heating produces subsequent melting of the ice at $\sim 260 \mathrm{~K}$. The observed melting point is $\sim 10 \mathrm{~K}$ lower than that of pure ice as a result of the colligative properties of the dissolved creatine and citric acid molecules.

Plots of the natural logarithm of dynamic viscosity ( In $\eta$ ) against the reciprocal of absolute temperature $(1 / T)$ are provided in Fig. 3 to classify the viscosity-temperature dependence of the water-containing 1:1 CrA:CAA system. It is notable that a Vogel-Fulcher-Tammann (VFT) model describes 
the data no better than a simple Arrhenius (linear) fit, despite having an additional fitting parameter (i.e., in all four cases shown, the correlation coefficient is essentially identical for Arrhenius and VFT fits). This demonstrates that these DESs do not deviate strongly from Arrhenius behaviour. Using linear least-squares fitting methods, the slope of the straight line is equal to $E_{\eta} / R$, allowing us to determine the activation energy for viscous flow $\left(E_{\eta}\right)$ values of $131,107,69.1$, and $61.3 \mathrm{~kJ} \mathrm{~mol}^{-1}$ for the 1:1 CrA:CAA system containing 50.0, 60.0, 66.7, and 71.4 mol\% water, respectively. The $E_{\eta}$ calculations provide a measure of how rapidly viscosity changes with temperature and these large values for $E_{\eta}$ are consistent with our previous observation that $E_{\eta}$ loosely correlates with ambient viscosity. ${ }^{9}$ Also shown in Fig. 3, as a benchmark for comparison, are the results for the DES glyceline which comprises 1:2 choline chloride:glycerol. The glyceline measurements yield an $E_{\eta}$ value of $42 \mathrm{~kJ} \mathrm{~mol}^{-1}$, in full agreeing with the literature value of $42.3 \mathrm{~kJ} \mathrm{~mol}^{-1} .{ }^{10} \mathrm{As}$ another point of reference, the viscosity activation energy for the "neat" DES reline (i.e., 1:2 choline chloride:urea) is reportedly $51.2 \mathrm{~kJ}$ $\mathrm{mol}^{-1} \cdot{ }^{9}$ Although not directly measurable using the available instrumentation, the $\eta(T)$ profiles for equimolar CrA:CAA are

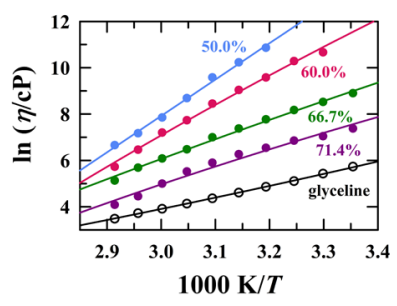

Fig. 3. Arrhenius plots of viscosity against reciprocal absolute temperature for the 1:1 creatine:citric acid deep eutectic system. The numbers shown indicate mol\% water. Results for the common DES glyceline (i.e., 1:1 choline chloride:glycerol) are provided as a foil for contrast.

reasonably well-described by exponential decays, allowing us to extrapolate viscosities of roughly 75,000 and $400,000 \mathrm{cP}$ at 298 $\mathrm{K}$ for 1:1 CrA:CAA containing 50.0 and 60.0 mol\% water, respectively (see Fig. S4 in the ESI). Given these trends, the viscosity for the anhydrous 1:1 CrA:CAA DES is projected to well exceed $10^{6} \mathrm{cP}$.

We next sought to estimate the micropolarity within the water-containing 1:1 CrA:CAA DES system. The DimrothReichardt $E_{\mathrm{T}}(30)$ empirical polarity scale based on the dye 2,6diphenyl-4-(2,4,6-triphenyl-1-pyridino)phenolate represents one of the most widely quoted measures of solvent polarity. Unfortunately, the carboxylic acid content of the 1:1 CrA:CAA system led to protonation of the basic phenoxide oxygen (dye $\left.\mathrm{p} K_{\mathrm{a}}=8.65\right)$, resulting in complete loss of the intramolecular $\pi-$ $\pi^{*}$ charge transfer absorption band used to determine the $E_{\mathrm{T}}(30)$ value. In fact, the much less basic dichloro analogue 2,6dichloro-4-(2,4,6-triphenyl-1-pyridinio)phenolate (Reichardt's dye $33, p K_{\mathrm{a}}=4.78$ ) also remained protonated (i.e., colourless), precluding its function as a solvatochromic indicator in the creatine:citric acid eutectic system.
The most widely applied methods for the determination of the Kamlet-Taft hydrogen bond donating acidity (alpha) depend on the knowledge of certain quantities, such as $E_{T}(30)$. The parameters that describe solvent dipolarity/polarizability $\left(\pi^{*}\right)$ and solvent hydrogen bond accepting basicity $(\beta)$ were determined ${ }^{11}$ for 1:1 CrA:CAA with varying water contents (Fig, 4a). The $\pi^{*}$ value is observed to increase marginally with water content, plateauing at a value of $1.28 \pm 0.01$ for $60.0 \mathrm{~mol} \%$ water and beyond. Interestingly, this suggests that the solvatochromic probe $\mathrm{N}, \mathrm{N}$-diethyl-4-nitroaniline, used to assess $\pi^{*}$ experiences an environment akin to water $\left(\pi^{*}=1.33\right)$ in these hydrated systems. This apparent dipolarity/polarizability is notably higher than that seen for the conventional reline, ethaline and glyceline DESs, all residing in the $\pi^{*}$ range from 1.14 to $1.23 .^{12}$ While the $\beta$ parameter similarly reaches a steady value $(0.33 \pm 0.01)$ at higher water contents, the eutectic system shows a stronger hydrogen bond basicity than water $(\beta=0.14)$.

The uncharged phenoxazone dye Nile Red (NR) is a useful polarity-sensitive fluorescent probe of hydrophobic environments. ${ }^{13}$ As shown in Fig. 4b, the NR absorbance maximum presents a small $(4-5 \mathrm{~nm})$ bathochromic shift as the water content in the system increases from 33.0 to $77.8 \mathrm{~mol} \%$. The molar transition energy value for NR calculated from the absorption band is associated with an $E_{\mathrm{NR}}$ value of $203.3 \pm 0.20$ $\mathrm{kJ} \mathrm{mol}^{-1}$ across the composition explored, a value approaching that of water $\left(201.7 \mathrm{~kJ} \mathrm{~mol}^{-1}\right)$. The corresponding fluorescence emission maximum change displayed as the water content was varied is substantial ( $28 \mathrm{~nm}$; Fig. S5), yielding a considerable increase in the Stokes shift $(\Delta E)$ for NR from $1,082 \mathrm{~cm}^{-1}$ at 33.0 mol\% water to $1,624 \mathrm{~cm}^{-1}$ at $77.8 \mathrm{~mol} \%$ water. For reference, the NR emission maxima for the 33.0 and 77.8 mol\% water samples (626 and $654 \mathrm{~nm}$ ) are analogous to the NR maxima in acetonitrile/DMF and ethylene glycol, respectively. ${ }^{13 a, 14}$
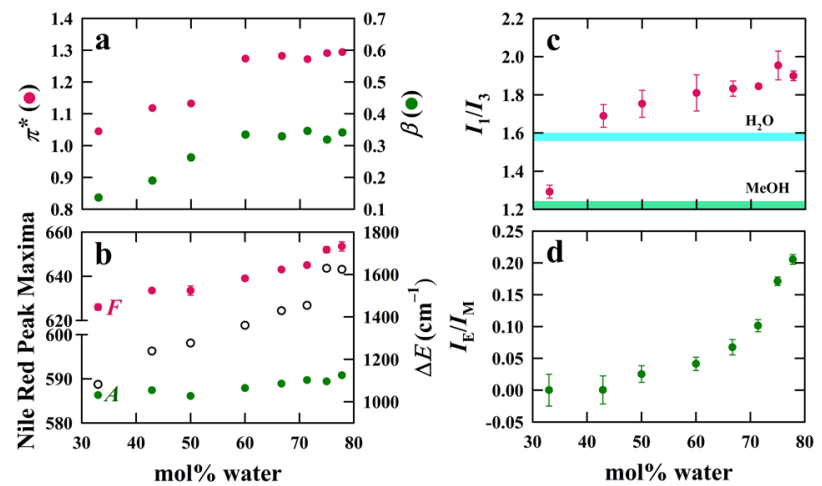

Fig. 4. Summary of solvatochromic probe studies. (a) Variation in Kamlet-Taft parameters $\beta$ and $\pi^{*}$ with mol\% water in the $1: 1$ creatine:citric acid system. The typical error is \pm 0.01 . (b) Nile Red absorbance $(A)$ and fluorescence $(F)$ emission maxima and calculated Stokes shift $(\Delta E)$ in the same eutectic system. (c) Experimental pyrene $I_{1} / I_{3}$ and (d) BPP $I_{E} / I_{M}$ intensity ratios for the binary creatine:citric acid eutectic system as functions of water content. Benchmark $I_{1} / I_{3}$ values are provided for water and methanol. 
Because the water content can be smoothly controlled over a significant range while retaining the key features of the eutectic mixture, we employed additional pyrene-based fluorescent probes to further characterize the creatine-citric acid system. Pyrene is a ubiquitous fluorescent probe of polarity within complex, organized media. The pyrene polarity scale, defined by the $I_{1} / I_{3}$ ratio describing the relative intensities of the vibronic bands of pyrene fluorescence at positions " 1 " (0-0 band, $373 \mathrm{~nm}$ ) and " 3 " ( 384 nm), is a function of the solvent dielectric $(\varepsilon)$ and refractive index $(n)$ via the dielectric cross term, $\left[f\left(\varepsilon, n^{2}\right)\right] \cdot{ }^{14}$ Fig. $4 c$ summarizes the pyrene $I_{1} / l_{3}$ intensity ratio measured for creatine:citric acid containing 33.0-77.8 mol\% water. Interestingly, the initial dipolarity at $33.0 \mathrm{~mol} \%$ water is akin to methanol, while the local dipolarity surrounding the average pyrene molecule increases with water content to become significantly more polar than water itself.

A final set of experiments was conducted to track the conformational flexibility of 1,3-bis(1-pyrenyl)propane (BPP) dissolved within the binary 1:1 CrA:CAA mixture with incremental water incorporation. Upon photoexcitation, BPP reorients to form an intramolecular excited-state dimer (excimer). ${ }^{16}$ The efficiency of excimer formation compared to the locally excited (monomer) state in this "two-state" mechanism can be followed by steady-state fluorescence. A summary of the relative extent of BPP excimer formation as a function of mol\% water in 1:1 CrA:CAA is provided in Fig. 4d where $I_{\mathrm{E}} / I_{\mathrm{M}}$ denotes the excimer-to-monomer fluorescence intensity ratio. At 33.0 mol\% water, excimer formation is completely arrested; however, as the water content increases, the excimer formation process is gradually switched on. The cyclization dynamics are essentially a report of the average, local fluidity or "microviscosity." Under similar experimental conditions, $I_{\mathrm{E}} / I_{\mathrm{M}} \approx 2$ for BPP in pure ethanol. This effectively shows the possibility for controlled chain dynamics to occur in the creatine:citric acid DES (a feature highly pertinent to polymerization, for example). The diffusion-controlled excimer formation process can be further modulated by temperature to achieve a wide range of conformational dynamics within this new eutectic system.

Dicyano-bis(1,10-phenanthroline)iron(II), whose absorption spectrum is sensitive to solvent-acceptor properties, was employed as a final probe. The wavelength of the absorption maximum in the visible region shows a hypsochromic shift as water content increases in the system (Fig. S6). Indeed, the Catalán solvent acidity correspondingly increases from 0.63 to 0.79 as the water content increases from 50.0 to $77.8 \mathrm{~mol} \%$ (Fig. S7), with a parallel shift in the Kamlet-Taft $\alpha$ value from 1.09 to 1.34 , the latter value exceeding that in neat water or acetic acid.

In conclusion, we have shown that the $\chi-T$ binary phase diagram of creatine and citric acid reveals a broad and stable deep eutectic region of variable composition. In addition, the phase diagram for the water-containing 1:1 binary creatine:citric acid system has been mapped out to establish the upper boundary for hydration whereafter the system stops behaving as a eutectic and devolves to an aqueous solution. Systematic studies of phase behavior can help discover new classes and compositions of DESs with desirable properties.
Solvatochromic probe studies demonstrate the ability to facilely control the microenvironmental polarity and molecular cyclization dynamics (microviscosity) within the creatine:citric acid system by managing water content. The ease of modulating viscosity and polarity within the biocompatible creatine:citric acid DES opens up broad avenues for biological applications.

\section{Notes and references}

1 a) Q. Zhang, K. De Oliveira Vigier, S. Royera, F. Jérôme, Chem. Rev., 2012, 41, 7108-7146; b) E. L. Smith, A. P. Abbott, K. S. Ryder, Chem. Rev., 2014, 114, 11060-11082; c) D. V. Wagle, H. Zhao, G. A. Baker, Acc. Chem. Res., 47, 2299-2308; d) B. B. Hansen, S. Spittle, B. Chen, D. Poe, Y. Zhang, J. M. Klein, A. Horton, L. Adhikari, T. Zelovich, B. W. Doherty, B. Gurkan, E. J. Maginn, A. Ragauskas, M. Dadmun, T. A. Zawodzinski, G. A. Baker, M. E. Tuckerman, R. F. Savinell, J. R. Sangoro, Chem. Rev., 2021, 121, 1232-128.

2 a) A. Paiva, R. Craveiro, I. Aroso, M. Martins, R. L. Reis, A. R. C. Duarte, ACS Sustainable Chem. Eng., 2014, 2, 1063-1071; b) Y. Liu, J. B. Friesen, J. B. McAlpine, D. C. Lankin, S.-N. Chen, G. F. Pauli, J. Nat. Prod., 2018, 81, 3, 679-690.

3 R. B. Kreider, Mol. Cell. Biochem., 2003, 244, 89-94.

4 K. B. Pekar, J. B. Lefton, C. A. McConville, J. Burleson, D. Sethio, E. Kraka, T. Runčevski, Cryst. Growth Des., 2021, 21, 1297-1306.

5 a) M. H. Shafie, R. Yusof, C.-Y.Gan, J. Mol. Liq., 2009, 288, $111081 ;$ b) Y. Guinet, L. Paccou, A. Hédoux, J. Mol. Liq., 2020, 318, 114317.

6 a) Q. Lu, G. Zografi, J. Pharm. Sci., 1997, 86, 1374-1378; b) J. Wang, R. Chang, Y. Zhao, J. Zhang, T. Zhang, Q. Fu, C. Chang, A. Zeng, AAPS PharmSciTech, 2017, 18, 2541-2550.

7 a) O. S. Hammond, D. T. Bowron, K. J. Edler, Angew. Chem. Int. Ed., 2017, 56, 9782-9785; b) A. Pandey, S. Pandey, J. Phys. Chem. B, 2014, 118, 14652-14661.

8 a) N. Yoshihara, M. Shibaya, H. Ishihara, J. Polymer Eng., 2005, 25, 97-114; b) A. Bogdan, M. J. Molina, H. Tenhu, T. Loerting, J. Phys. Chem. A, 2015, 119, 4515-4523.

9 H. Jin, B. O'Hare, J. Dong, S. Arzhantsev, G. A. Baker, J. F. Wishart, A. J. Benesi and M. Maroncelli, J. Phys. Chem. B, 2008, 112, 81-92.

10 G. Gygli, X. Xu and J. Pleiss, Sci. Rep., 2020, 10, 21395.

11 S. N. Baker, G. A. Baker and F. V. Bright, Green Chem., 2002, 4, 165-169.

12 S. Trivedi, N. I. Malek, K. Behera and S. Pandey, J. Phys. Chem. B, 2010, 114, 8118-8125.

13 a) D. L. Sackett and J. Wolff, Anal. Biochem, 1987, 167, 228-234; b) K. A. Fletcher, I. A. Storey, A. E. Hendricks, S. Pandey and S. Pandey, Green Chem., 2001, 3, 210-215.

14 A. Pandey, R. Rai, M. Pal and S. Pandey, Phys. Chem. Chem. Phys., 2014, 16, 1559-1568.

15 a) D. S. Karpovich and G. J. Blanchard, J. Phys. Chem., 1995, 99, 3951-3958; b) D. C. Dong and M. A. Winnik, Can. J. Chem., 1984, 62, 2560-2565.

16 a) G. A. Baker, S. N. Baker and T. M. McCleskey, Chem. Commun., 2003, 2932-2933; b) A. Sarkar, M. Ali, G. A. Baker, S. Y. Tetin, Q. Ruan and S. Pandey, J. Phys. Chem. B, 2009, 113, 3088-3098; c) K. A. Fletcher, S. N. Baker, G. A. Baker and S. Pandey, New J. Chem., 2003, 27, 1706-1712. 